\title{
Request for Immediate Intervention by the Public Health Authorities to Counter the Infodemic Related to the Russian Attack in Ukraine
}

Author: Alessandro Rovetta

Affiliation: R\&C Research, Bovezzo (BS), Italy

ORCID: http://orcid.org/0000-0002-4634-279X

Email: rovetta.mresearch@gmail.com

Phone: +393927112808

As pointed out by the WHO director Tedros Adhanom Ghebreyesus, during the COVID-19 global health crisis, the world had to face not just an epidemic but also a colossal infodemic. The excess of fake news is capable of causing an unnecessary increase in harmful and irrational feelings such as fear, terror, confusion, anxiety, stress, and depression, or, conversely, inadequately low risk perception. The former can seriously compromise individuals' mental health while the latter their - and others' - physical health [1]. Indeed, conspiracy hypotheses push people to engage in inappropriate behaviors (e.g., the assumption of dangerous substances) or not comply with safeguard regulations (e.g., vaccine hesitancy). Even numerous mass media and scientists have been guilty of aggravating this phenomenon, often providing compelling information or outright disinformation [2]. Furthermore, the population has already fronted pandemic fatigue resulting from two emotionally challenging years. In this regard, in Italy - one of the countries most affected by COVID-19 - there has been an alarming increase in the nonadherence to anti-contagion measures, even going so far as to buy or make counterfeit green passes [3].

Sadly, the current war unleashed by the Russian government against Ukraine, besides causing unjustifiable suffering and death, is generating a new war-related infodemic. In particular, our rapid analysis using the Google Trends infodemiological tool revealed a drastic growth in keywords linked not only to war but also to possible anti-nuclear remedies such as bunkers and iodine tablets. Comparing the relative search volumes of the "war, radioactive + radioactivity + nuclear + atomic + atomic, bunker (topic), and iodine" keywords for the periods 24 February - 2 March 2022 and 5 December 2021 - 23 February 2022 (last 90 days), we observed an average increase of $1700 \%, 980 \%, 1600 \%$, and $200 \%$ respectively (Mann-Whitney $U$ test P-values<.001, Figure 1). Looking at the data of the previous five years, no trend or seasonality capable of justifying this upsurge has been highlighted. Hence, the dread is real and exponentially growing, setting the stage for a massive new wave of dis-misinformation. These dynamics faithfully recall the first COVID-19 wave, when people sought fast remedies to relieve tension, often stumbling upon disinformation websites [4]. Moreover, we again risk witnessing the emergence of stigma, segregation, and racism towards the Russian people, just as it happened with the Chinese people [2]. In this case, it is essential to emphasize that the Russian political situation is a dictatorship, and it is unfair to unconditionally condemn a population that does not have the right to express its thoughts and condolences freely [5]. The policy of isolation against Russia is adequate, but the goal must be solely to oppose the government so as to defend the Ukrainian population. Besides, we observed the main search topics and queries suggested by Google Trends without entering any keywords from February 24 (the first 
Russian attack in Ukraine) onwards. In this way, we let Google Trends suggest the topics of greatest interest on the web in Italy. Once again, we have confirmed that the interest in the war was extremely high (21/25 topics) and included searches such as "Nuclear weapon" (rising topic, $+2800 \%$ ). Users frequently search for conflict news, which can easily lead to fake news reading. The accredited anti-hoax website "Butac.it" - known for having been adopted in various infodemiological investigations and for collaborating with the Italian National Federation of Orders of Physicians and Dentists [3] - reported that the same sources of COVID-19 disinformation are now active about the conflict [5]. For all these reasons, we strongly suggest that government authorities implement adequate information strategies and use Google Trends to quickly monitor citizens' war-related web searches and topics of growing hype in order to intervene promptly and in a targeted manner.

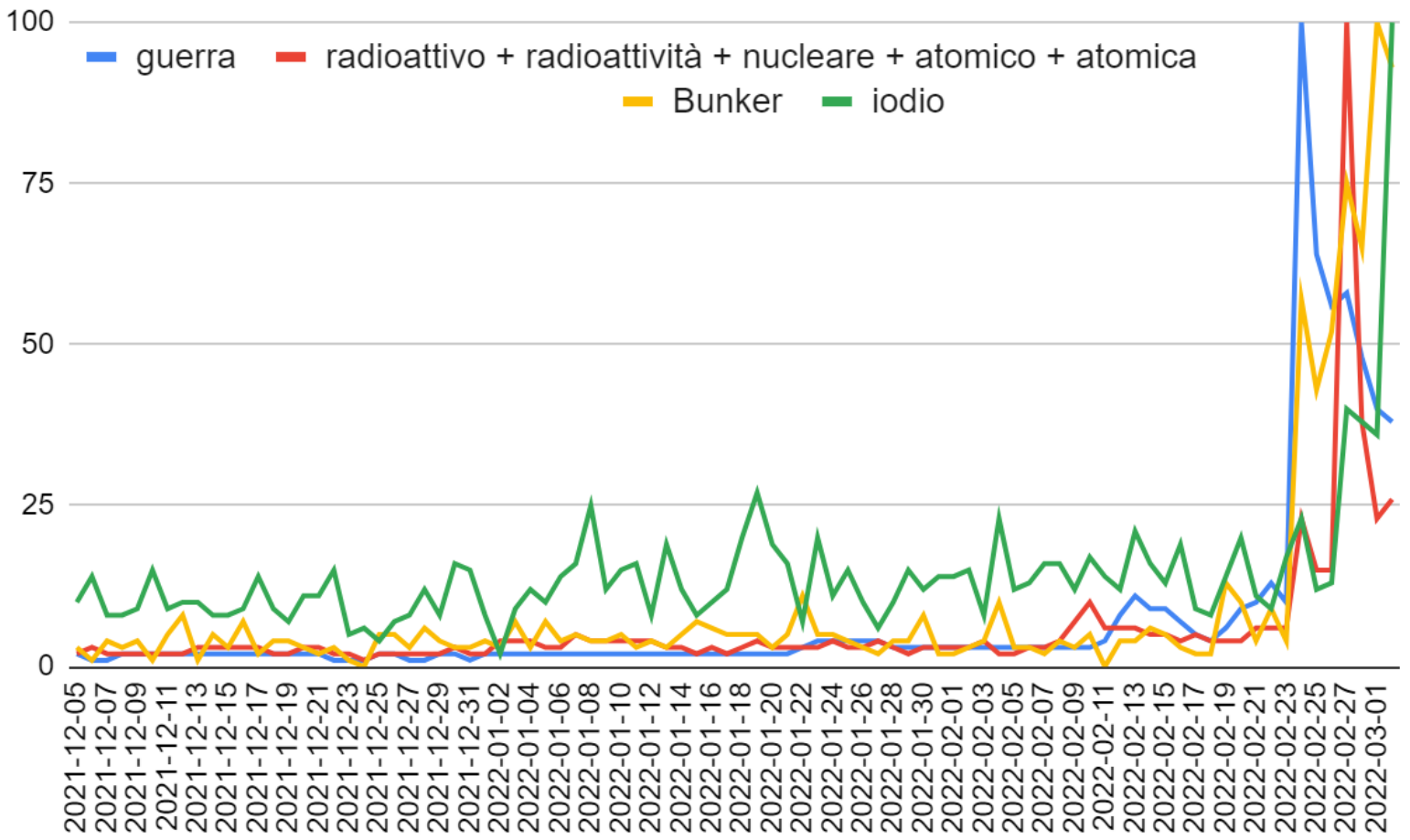

Figure 1. Italian netizens' web search volumes related to war (blue), nuclear (red), bunker (yellow), and iodine (green) from December 2021 to early March 2022. 


\section{References}

[1] Infodemic. World Health Organization. Accessed 2022, Mar 05 . URL: https://www.who.int/health-topics/infodemic

[2] Rovetta A, Castaldo L. A new infodemiological approach through Google Trends: longitudinal analysis of COVID-19 scientific and infodemic names in Italy. BMC Med Res Methodol. 2022 Jan 30;22(1):33. doi: 10.1186/s12874-022-01523-x. PMID: 35094682; PMCID: PMC8801192.

[3] Rovetta A. Infodemic Emergency in Italy: A Longitudinal Analysis of the Web Interest in Sources of Dis-misinformation, Epidemiologically Dangerous Behaviors, and Vaccine Hesitancy During COVID-19. SocArXiv preprint. 2022, Feb 10. doi:10.31235/osf.io/bu9tx.

[4] Fake News. Ministero della Salute. Accessed 2022, Mar 05. URL: https://www.salute.gov.it/portale/nuovocoronavirus/archivioFakeNewsNuovoCoronavirus.jsp

[5] Screditare i media. Butac.it. Accessed 2022, Mar $05 . \quad$ URL: https://www.butac.it/screditare-i-media/ 